\title{
Numerical study of jets produced by conical wire arrays on the Magpie pulsed power generator
}

\author{
Matteo Bocchi ${ }^{1}$, Jerry P. Chittenden ${ }^{1}$, Andrea Ciardi ${ }^{2,3}$, \\ Francisco Suzuki-Vidal ${ }^{1}$, Gareth N. Hall ${ }^{1}$, Phil de Grouchy ${ }^{1}$, \\ Sergei V. Lebedev ${ }^{1}$, Simon C. Bott ${ }^{4}$ \\ ${ }^{1}$ The Blackett Laboratory, Imperial College London, SW7 2BW London, UK \\ email: m.bocchi@imperial.ac.uk \\ ${ }^{2}$ LERMA, Université Pierre et Marie Curie, Observatoire de Paris, Meudon, France \\ ${ }^{3}$ École Normale Supérieure, Paris, France. UMR 8112 CNRS \\ ${ }^{4}$ Center for Energy Research, University of California, San Diego, CA, USA
}

\begin{abstract}
With the aim to model jets produced by conical wire arrays on the MAGPIE generator, and to strengthen the link between laboratory and astrophysical jets, we performed three-dimensional magneto-hydro-dynamic numerical simulations using the code GORGON and successfully reproduced the experiments. We found that a minimum resolution of $\sim 100 \mu \mathrm{m}$ is required to retrieve the unstable character of the jet. Moreover, arrays with less wires produce more unstable jets with stronger magnetic fields around them.
\end{abstract}

Keywords. ISM: jets and outflows, hydrodynamics, plasmas, methods: laboratory, methods: numerical

\section{Introduction}

Important improvements have been achieved in the comprehension of astrophysical jets. However, several questions remain open, including jet formation, propagation in an external medium and survival to potentially disruptive instabilities (Bellan et al., 2009; Hardee, 2004). Jets produced by conical wire arrays are characterised by dimensionless parameters in a similar range to Young Stellar Object (YSO) jets, and are especially suitable to study the interaction of the jet with an ambient medium (Lebedev et al., 2005; Ciardi et al., 2008). We produced laboratory jets on the MAGPIE pulsed power generator at Imperial College, London (Mitchell et al., 1996) using different setups: conical and radial wire arrays (Lebedev et al., 2005) and radial foils (Ciardi et al., 2009). New physical regimes for jets are possible after the recent upgrade of MAGPIE and the "Z" pulsed power facility in Sandia, USA. Our aim is to model the conical wire array experiments on MAGPIE to understand the physics and help in the design of new diagnostics and experimental setups. The three-dimensional (3D) resistive magneto-hydro-dynamic (MHD) code GORGON (Chittenden et al., 2004; Ciardi et al., 2007) is used for the modelling.

\section{Model and numerical setup}

GORGON (Chittenden et al., 2004; Ciardi et al., 2007) is a fully resistive, 3D MHD code based on the Van Leer algorithm. Ions and electrons are treated as a single fluid. However, the energy equations are solved separately. The vector potential $\mathbf{A}$ is diffused and advected in order to follow the electromagnetic fields. The code includes optically 

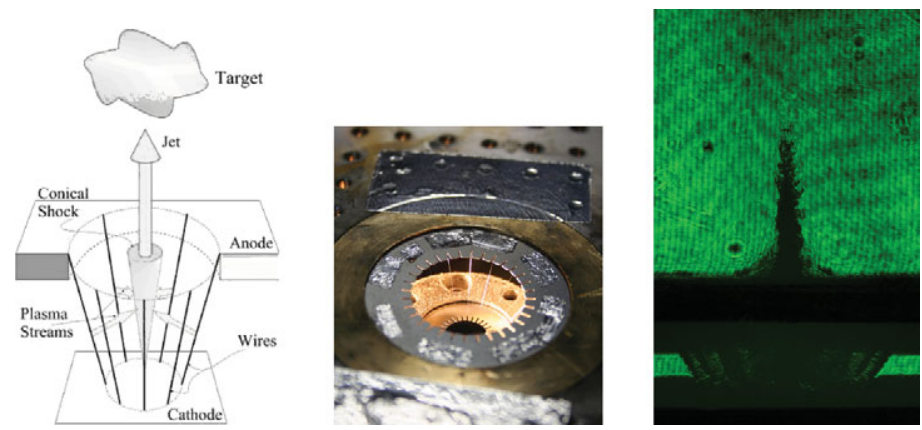

Figure 1. Left Panel: Scheme of a typical conical array setup. Central Panel: Picture of the experimental setup. Right Panel: Laser shadowgram of the shot $s 0301 \_10$ taken at $355 \mathrm{~ns}$. Array of 16 tungsten wires of $18 \mu \mathrm{m}$ diameter.

thin radiation losses and Ohmic heating, as well as a "computational vacuum" below a density $\rho_{v a c}=10^{-4} \mathrm{Kg} / \mathrm{m}^{3}$, in which only the wave equation for $\mathbf{A}$ is solved.

A conical wire array is a set of wires in a conical shape, passed through by a high current pulse (see Fig. 1). The current heats the wires transforming them into plasma, and produces at the same time a global toroidal magnetic field which accelerates the plasma towards the axis of the array in a direction normal to the wires. Around the axis, a conical shock is formed. The component of the velocity parallel to the axis is conserved across the shock, so that a mainly hydrodynamic, collimated jet is formed.

We chose the numerical setup following the recent experiments carried out on the MAGPIE facility in London. In our simplified setup, two electrode plates are connected by a variable number of equally spaced wires, as seen in Fig. 1. In our reference case the cathode diameter is $d_{c}=12 \mathrm{~mm}$, the anode diameter is $d_{a}=21 \mathrm{~mm}$, and the array length (the distance between cathode and anode) is $l=12 \mathrm{~mm}$. The inclination angle is $\sim 30$ degrees. We simulated arrays with 16 and 32 tungsten wires. The electric current provided by the MAGPIE generator has been modeled using the following expression:

$$
I(t)=I_{0} \cdot \sin ^{2}(\pi t / 2 \tau),
$$

where $I_{0}=1.4 \cdot 10^{6}$ is the peak current in Ampere and $\tau=250 \cdot 10^{-9}$ is the peak time in seconds. The resolution employed was either 100 or $200 \mu \mathrm{m}$.

\section{Results}

Our simulations successfully reproduced the general behaviour observed in the experiments. The jet forms along the axis and displays an unstable, turbulent character in agreement with the observations (see Fig. 1, right panel). In what follows we present the differences arising when changing the number of wires and resolution, visible in Fig. 2.

First, we note that the jet appears to be turbulent only in the high resolution simulations. The non-axisimmetric features particularly visible in the case with 16 wires are not found in simulations with the low resolution of $200 \mu \mathrm{m}$. The profiles of the various physical quantities along the jet axis (not shown) are remarkably similar for the two different resolution employed, despite the big difference in the level of turbulence. In particular, velocity and temperature are compatible, while the density is systematically lower in the low resolution case. However, the general trend of the physical variables is preserved. We found that a minimum resolution of about $100 \mu \mathrm{m}$ is needed to accurately reproduce the laboratory results. Indeed, the surface of the jet must be resolved numerically with enough grid points to properly follow the steep density gradients. 

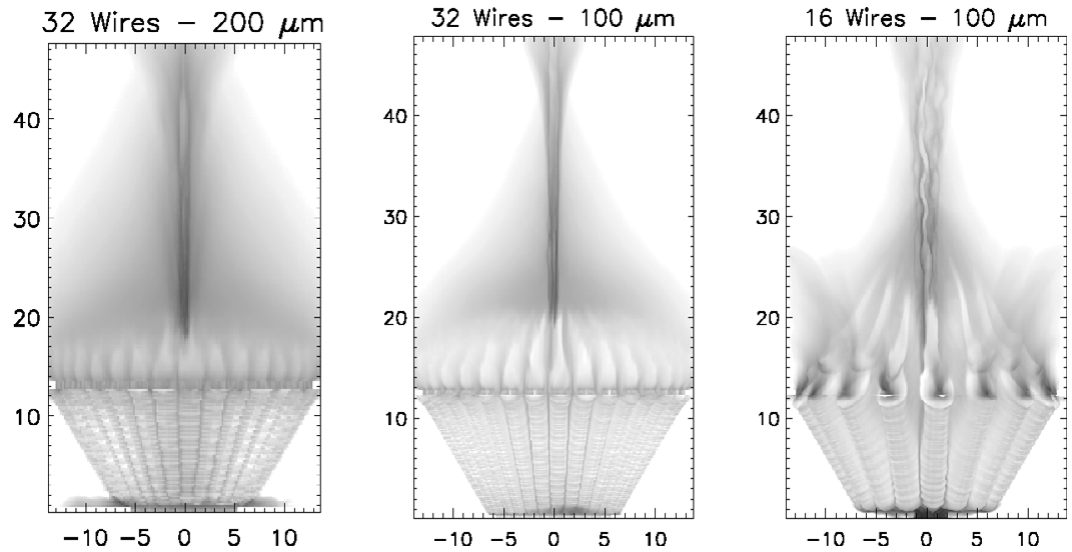

Figure 2. Emission maps of three different simulations at 400 ns. Darker shades correspond to higher emission. The axis are in $\mathrm{mm}$ units. Left Panel: 32 wires, $200 \mu \mathrm{m}$ resolution. Central Panel: 32 wires, $100 \mu \mathrm{m}$ resolution. Right Panel: 16 wires, $100 \mu \mathrm{m}$ resolution.
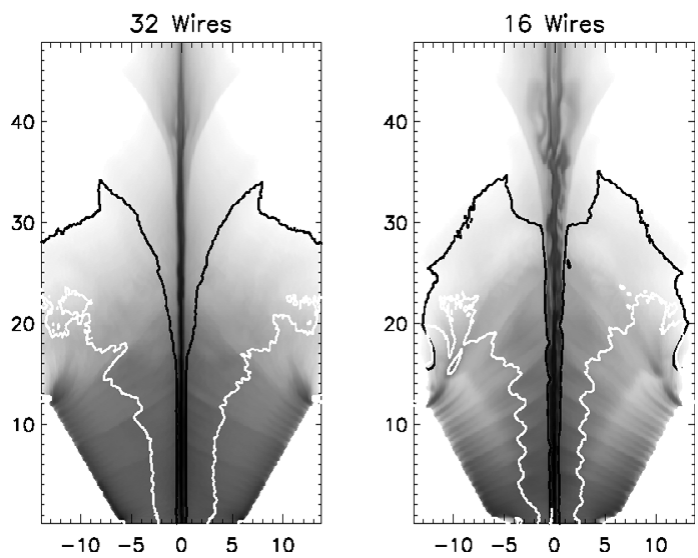

Figure 3. Density cuts in the mid-plane of two different simulations at 350 ns. Darker shades correspond to higher values. The axis are in $\mathrm{mm}$ units. Left Panel: 32 wires, $100 \mu \mathrm{m}$ resolution. Right Panel: 16 wires, $100 \mu \mathrm{m}$ resolution. Black contours represent the plasma beta: $\beta=1$, higher values towards the axis. White contours represent the kinetic plasma beta (see text): $\beta_{k}=10$, higher values towards the axis.

Second, the jet produced by the 16 wires array appears to be much more unstable than the one in the 32 wires array (see Fig. 3). A comparison of the magnetic field strength reveals that the field is much stronger in the 16 wires case, as it can penetrate more easily in the larger inter-wire gaps. The plasma beta (thermal over magnetic pressure), black contours in Fig. 3, seems to show that the magnetic field dominates in the region just around the jet. However, from the kinetic plasma beta (kinetic ram pressure over magnetic pressure), white contours, it is clear that the jet is kinetically dominated. The reasons for higher instability of the 16 wires simulations are not completely understood, but can be sought in the greater density modulations in the plasma flowing from the wires, visible in Fig. 3. Moreover, less wires produce less streams meeting on axis to form the jet, therefore reducing the overall symmetry of the system. 


\section{Conclusions}

In this paper we presented the results of our numerical investigation on the jets produced by conical wire arrays on the pulsed power generator MAGPIE in London.

First, only a resolution of about $\sim 100 \mu \mathrm{m}$ proved to be sufficient to reproduce the jet unstable character observed in the experiments. Lower resolution simulations maintained the general trend of the physical variables to some extent, but could not resolve the details in the jet.

Second, higher wire numbers mean smaller inter-wire gaps, therefore a much smoother distribution of the magnetic field and density. In the case of large inter-wire gaps however, fields can penetrate deeper in the array and are stronger around the jet. Nevertheless, the jet is kinetically dominated. Moreover, the density distribution is less regular, enhancing the unstable character of the jet.

Future work will consider, among other parameters, the effect of the inclination angle on the resulting jet. We will support the study with new sets of experiments both on MAGPIE in London and on the "Z" generator in Sandia, USA. We also plan experiments of the interaction of the jet with a gas target.

Our final goal is to connect experiments and simulations with the astrophysical scenario. YSO jets form in and propagate through molecular clouds. The subsequent interaction is mostly recognizable in the bow shock at the jet head, but is also responsible for material entrainment from the surrounding medium. The morphology and dynamics of these regions are of great interest for the study of chemical evolution and turbulent behaviour of molecular clouds. Studying such aspects in the laboratory is now possible, taking advantage of the accurate diagnostics available to plasma physicists. Therefore, laboratory jets provide very useful information to understand astrophysical jets.

\section{Acknowledgements}

Work supported by the EPSRC Grant No. EP/G001324/1 and by the NNSA under DOE Cooperative Agreements No. DE-F03-02NA00057 and No. DE-SC-0001063. Simulations were also run on the Jade supercomputer (GENCI-CINES, Paris-Montpellier, France), with support of the HPC-Europa2 project funded by the European Commission - DG Research in the Seventh Framework Programme under grant agreement No.228398.

\section{References}

Bellan, P. M., Livio, M., Kato, Y., Lebedev, S. V., Ray, T. P., Ferrari, A., Hartigan, P., Frank, A., Foster, J. M., \& Nicolaï, P. 2009, Physics of Plasmas, 16, 041005

Hardee, P. E. 2004, Astrophysics and Space Science, 293, 117

Lebedev, S. V., Ciardi, A., Ampleford, D. J., Bland, S. N., Bott, S. C., Chittenden, J. P., Hall, G. N., Rapley, J., Jennings, C., Sherlock, M., Frank, A., \& Blackman, E. G. 2005, Plasma Phys. Control. Fusion, 47, B465

Ciardi A., Ampleford, D. J., Lebedev, S. V., \& Stehle, C. 2008, ApJ, 678, 968

Mitchell, I. H., Bayley, J. M., Chittenden, J. P., Worley, J. F., Dangor, A. E., Haines, M. G., \& Choi, P. 1996, Rev. Sci. Instrum., 67, 1533

Ciardi, A., Lebedev, S. V., Frank, A., Suzuki-Vidal, F., Hall, G. N., Bland, S. N., HarveyThompson, A., Blackman, E. G., \& Camenzind, M. 2009, ApJ, 691, L147

Chittenden, J. P., Lebedev, S. V., Jennings, C., Bland, S. N., \& Ciardi, A. 2004, Plasma Phys. Control. Fusion, 46, B457

Ciardi, A., Chittenden, J. P., Lebedev, S. V., Bland, S. N., Bott, S. C., Rapley, J., Hall, G. N., Suzuki-Vidal, F., Marocchino, A., Lery, T., \& Stehle, C. 2007, Physics of Plasmas, 14, 056501 